\title{
Increased Neural Activity in Mesostriatal Regions after Prefrontal Transcranial Direct Current Stimulation and L-DOPA Administration
}

\author{
Benjamin Meyer, ${ }^{1,2}$ @Caroline Mann, ${ }^{1,3}$ Manuela Götz, ${ }^{1,2}{ }^{\oplus}$ Anna Gerlicher, ${ }^{1,2}$ Victor Saase, ${ }^{1}$ Kenneth S.L. Yuen, ${ }^{1,2}$ \\ Felipe Aedo-Jury, ${ }^{2,4}$ @ Gabriel Gonzalez-Escamilla, ${ }^{5}$ Albrecht Stroh, ${ }^{2,4}$ and Raffael Kalisch ${ }^{1,2}$ \\ ${ }^{1}$ Neuroimaging Center (NIC), Focus Program Translational Neuroscience, ${ }^{2}$ Deutsches Resilienz Zentrum (DRZ), Johannes Gutenberg University Medical \\ Center Mainz, 55131 Mainz, Germany, ${ }^{3}$ Department of Child and Adolescent Psychiatry, Psychosomatics and Psychotherapy, University Hospital \\ Frankfurt am Main, Goethe University Frankfurt am Main, 60590 Frankfurt am Main, Germany, ${ }^{4}$ Institute for Microscopic Anatomy and \\ Neurobiology, Focus Program Translational Neurosciences, and 5 Department of Neurology, Johannes Gutenberg University Medical Center Mainz, \\ 55131 Mainz, Germany
}

\begin{abstract}
Dopamine dysfunction is associated with a wide range of neuropsychiatric disorders commonly treated pharmacologically or invasively. Recent studies provide evidence for a nonpharmacological and noninvasive alternative that allows similar manipulation of the dopaminergic system: transcranial direct current stimulation (tDCS). In rodents, tDCS has been shown to increase neural activity in subcortical parts of the dopaminergic system, and recent studies in humans provide evidence that tDCS over prefrontal regions induces striatal dopamine release and affects reward-related behavior. Based on these findings, we used fMRI in healthy human participants and measured the fractional amplitude of low-frequency fluctuations to assess spontaneous neural activity strength in regions of the mesostriatal dopamine system before and after $\mathrm{tDCS}$ over prefrontal regions ( $n=40,22$ females). In a second study, we examined the effect of a single dose of the dopamine precursor levodopa (L-DOPA) on mesostriatal fractional amplitude of low-frequency fluctuation values in male humans $(n=22)$ and compared the results between both studies. We found that prefrontal tDCS and L-DOPA both enhance neural activity in core regions of the dopaminergic system and show similar subcortical activation patterns. We furthermore assessed the spatial similarity of whole-brain statistical parametric maps, indicating tDCS- and L-DOPA-induced activation, and $>100$ neuronal receptor gene expression maps based on transcriptional data from the Allen Institute for Brain Science. In line with a specific activation of the dopaminergic system, we found that both interventions predominantly activated regions with high expression levels of the dopamine receptors D2 and D3.
\end{abstract}

Key words: dopamine; fALFF; fMRI; L-DOPA; resting state; tDCS

\section{Significance Statement}

Studies in animals and humans provide evidence that transcranial direct current stimulation (tDCS) allows a manipulation of the dopaminergic system. Based on these findings, we used fMRI to assess changes in spontaneous neural activity strength in the human dopaminergic system after prefrontal tDCS compared with the administration of the dopamine precursor and standard anti-Parkinson drug levodopa (L-DOPA). We found that prefrontal tDCS and L-DOPA both enhance neural activity in core regions of the dopaminergic system and show similar subcortical activation patterns. Using whole-brain transcriptional data of $>100$ neuronal receptor genes, we found that both interventions specifically activated regions with high expression levels of the dopamine receptors $\mathrm{D} 2$ and $\mathrm{D} 3$.

\section{Introduction}

The functional diversity of the dopaminergic system explains the close relationship between dopamine dysfunctions and a multi- faceted range of neuropsychiatric disorders, including Parkinson's disease (PD), addiction, and schizophrenia (Kalivas and Volkow, 2005; Goto and Grace, 2007; Galvan and Wichmann, 
2008). Thus, therapeutic strategies for manipulating dopaminergic system activity are of great clinical relevance, but, despite major advances, they are frequently accompanied by sometimes severe side effects (Katzenschlager and Lees, 2002; Foster and Hoffer, 2004; Appleby et al., 2007).

There is now increasing evidence from studies in animals and humans that transcranial direct current stimulation (tDCS) might be an effective nonpharmacological and noninvasive way to activate deep brain regions of the dopaminergic system. tDCS is a form of subthreshold brain stimulation that is based on a weak constant current, applied between an anodal and a cathodal electrode both placed on the scalp. Anodal stimulation causes the resting membrane potential to become slightly more positive, whereas cathodal stimulation slightly hyperpolarizes the membrane. Hence, rather than causing neurons to fire, tDCS is supposed to modulate their excitability (Nitsche and Paulus, 2000; Rahman et al., 2013).

Takano et al. (2011) combined tDCS with fMRI in rats and observed increased fMRI signal intensities in the NAcc after anodal stimulation over the frontal cortex. Using the same electrode placement, Leffa et al. (2016) found elevated striatal dopamine levels after tDCS. Moreover, Lu et al. (2015) showed that anodal tDCS over the frontal cortex not only increased whole-brain dopamine levels but also relieved symptoms in a mouse model of PD, comparable in effect with L-DOPA, a standard anti-PD drug, which is converted to dopamine in the intracellular space of dopaminergic midbrain neurons (Volkow et al., 1996).

Several studies in humans have already demonstrated effects on neural activity in striatal areas during and after tDCS over prefrontal and motor cortical areas (Polanía et al., 2012; Chib et al., 2013; Hone-Blanchet et al., 2016), and a recent study provided first molecular evidence of elevated striatal dopamine levels after prefrontal tDCS in humans (Fonteneau et al., 2018). Based on these results, we examined the enhancement of neural activity in regions of the dopaminergic system before and after prefrontal tDCS and compared the effect to a pharmacological stimulation of dopamine synthesis.

We conducted two separate resting-state fMRI (rsfMRI) studies in healthy humans. In the first study (tDCS study), we applied a tDCS protocol developed by Chib et al. (2013), who provided the first evidence for a causal manipulation of distant dopaminergic brain regions and associated dopamine-dependent functions after anodal stimulation over the frontopolar/ventromedial PFC (fp/vmPFC, 10-20 electrode system: Fpz) and cathodal stimulation over the right dorsolateral PFC (dlPFC, F4). In a parallel study design, we used the anodal/cathodal Fpz/F4 montage in the experimental group (main, $n=20$ ) and the same electrode locations but inverse polarity in a control group (inverse, $n=20$ ). We examined the fractional amplitudes of lowfrequency signal fluctuations (fALFFs), a proxy of spontaneous neural activity strength (Zou et al., 2008), in subcortical regions of the dopaminergic system before and after prefrontal tDCS. The same analysis was performed in a second study (L-DOPA study, $n=22$ ), in which the effect of a single dose of L-DOPA versus placebo was examined in a crossover design.

The effect of tDCS and L-DOPA on FALFF was examined in a dopaminergic system mask and fALFF changes in predefined subcortical regions (subcortical activation profiles) were com-

The authors declare no competing financial interests.

Correspondence should be addressed to Benjamin Meyer at benmeyer@uni-mainz.de.

https://doi.org/10.1523/JNEUROSCI.3128-18.2019

Copyright $\odot 2019$ the authors pared between both interventions. We furthermore compared tDCS- and L-DOPA-induced whole-brain activity patterns with $>100$ neuronal receptor gene expression maps, based on transcriptional data from the Allen Institute for Brain Science (AIB), to assess similarities between the interventions in receptorspecific activation patterns and to analyze the relative specificity for regions with high dopamine receptor expression.

To support L-DOPA-induced fALFF changes in the dopaminergic system from a cross-species perspective, we also report results from a study in which we analyzed fALFF in the dopaminergic system of medetomidine-sedated rats $(n=6)$.

\section{Materials and Methods}

\section{Experimental design and statistical analysis}

Procedures tDCS study (humans). A total of 42 healthy participants were enrolled. The Ethics Committee of the State Medical Board (RheinlandPfalz, Germany) approved the study, and all participants gave written informed consent. Regular use of illegal drugs was an exclusion criterion. Two participants were excluded due to technical problems $(n=40)$.

In a single-blind parallel study design, participants were randomly assigned to the main ( $n=20,11$ females, mean age: 25.7 years, age range: 21-32 years) or the inverse group ( $n=20,11$ females, mean age: 25.1 years, age range: $19-32$ years). There was no significant age difference between the groups $\left(t_{(38)}=0.57, p=0.573\right.$, two-tailed $t$ test $)$. rsfMRI data were acquired before (pre) and $\sim 5$ min after (post) tDCS application. Before the first rsfMRI measurement, electrode positions were marked on the participant's head to allow a fast electrode placement after the first scan. The 10-20 international system for electroencephalography was used for electrode positioning. We used a tDCS protocol developed by Chib et al. (2013) and placed a $3.5 \mathrm{~cm} \times 3.5 \mathrm{~cm}\left(12.25 \mathrm{~cm}^{2}\right)$ anode with its center over electrode position Fpz and a $5 \mathrm{~cm} \times 5 \mathrm{~cm}(25$ $\mathrm{cm}^{2}$ ) cathode over electrode position F4 in the main group. Using this specific protocol, Chib et al. (2013) were able to modulate frontomidbrain interactions and reported a correlation between tDCS-induced neural effects and reward-related behavioral changes. Hence, the authors found evidence for a causal manipulation of distant dopaminergic brain regions and associated dopamine-dependent functions. For the control condition, we also followed Chib et al. (2013), who, after an extensive series of testing, selected an active control condition with maximum similarity to the experimental condition in which the same electrode placement was used with inverse polarity. The electric field distribution was simulated using the SimNIBS software package (Thielscher et al., 2015).

tDCS was applied using a battery-driven constant-current stimulator (DC-Stimulator, neuroConn). Constant current was delivered for 15 min at $2 \mathrm{~mA}$ intensity ( $20 \mathrm{~s}$ ramp in and $20 \mathrm{~s}$ ramp out) through conductive rubber electrodes inserted into saline-soaked sponge pockets. Controlled by the DC-Stimulator, the impedance was kept $<10 \mathrm{k} \Omega$.

Procedures L-DOPA study (humans). A total of 24 healthy male participants were enrolled. Participation was restricted to male participants because of potential estrogen-dopamine interaction effects on brain activity (Sánchez et al., 2012). A board-certified physician screened participants for contraindications of L-DOPA intake. Participants who reported to take illegal drugs on a regular basis were excluded. Abuse of illegal drugs was tested by urine drug screen (M10/3-DT; Diagnostik Nord). Participants were asked about their smoking habits, but only 3 participants were smokers (each $<5$ cigarettes/d). The Ethics Committee of the State Medical Board (Rheinland-Pfalz, Germany) approved the study, and all participants gave written informed consent. Two participants were excluded from rsfMRI analyses due to head motion and severe tiredness. Head motion was assessed based on realignment parameters (see rsfMRI data preprocessing (humans)), and tiredness was assessed by monitoring the right eye of the participant using an MRI-compatible camera (MR Cam Model 12M; MRC Systems). Participants who closed their eyes continuously or repeatedly for more than $\sim 5 \mathrm{~s}$ during rsfMRI scans were excluded. Eventually, rsfMRI data from 22 participants were analyzed (mean age: 29.3 years, age range: $25-39$ years). 
rsfMRI data were acquired on 2 measurement days (days 1 and 2), separated by at least 5 and not $>14 \mathrm{~d}$ (Damoiseaux et al., 2006). In a crossover design, each participant received either L-DOPA at day 1 followed by a placebo treatment at day 2 or vice versa. A third person randomly assigned participants to one of the two treatment sequences. Experimenter and participants were both blinded. Participants were told not to eat $1.5 \mathrm{~h}$ before the L-DOPA/placebo intake. Drugs were administered orally as capsules of $150 \mathrm{mg}$ of L-DOPA with $37.5 \mathrm{mg}$ benserazide (Levodopa-Benserazid-ratiopharm) or an identically looking placebo capsule filled with mannitol and Aerosil. Drugs were prepared and provided by the pharmacy of the University Medical Center Mainz. On both days, L-DOPA/placebo administration was directly followed by an rsfMRI baseline measurement at which no L-DOPA effect can be expected $\left(\mathrm{LD}_{0 \text { min }} / \mathrm{Plc}_{0 \text { min }}\right)$. Further rsfMRI scans were performed after $45\left(\mathrm{LD}_{45} \mathrm{~min} /\right.$ $\left.\mathrm{Plc}_{45 \min }\right)$ and $90 \mathrm{~min}\left(\mathrm{LD}_{90 \mathrm{~min}} / \mathrm{Plc}_{90 \text { min }}\right)$, to capture the approximate times of maximum L-DOPA plasma concentration (Benetello et al., 1997; Hilal-Dandan and Brunton, 2014). Comparable time points have been chosen in other studies examining the effect of L-DOPA on resting-state activity (Flodin et al., 2012; Cole et al., 2013; Haaker et al., 2013). Participants stayed under medical observation for the duration of the experiment, including heart rate and blood pressure measurements and questionnaires on potential side effects.

Procedures L-DOPA study (rats). In an additional animal rsfMRI study, we tested the effect of L-DOPA on mesostriatal fALFF values in rats. Female Lewis rats $(n=6 ;>12$ weeks old; $160-180 \mathrm{~g})$ were used in this experiment due to their limited growth compared with males. Each animal was scanned under placebo $(\mathrm{NaCl}, 0.9 \%)$ and L-DOPA. The order was randomized, and the time interval between both measurements was at least 1 week. Animals were anesthetized with isoflurane $1.5 \%$ (Forene, Abbott) during the scanner placement procedure. Temperature and breathing rate were monitored during the entire experiment by an MRIcompatible monitoring system (SA Instruments). After the placement of the animal and the intraperitoneal injection of L-DOPA $(10 \mathrm{mg} /$ $\mathrm{kg})+$ benzeraside $(20 \mathrm{mg} / \mathrm{kg})$ or placebo, a bolus of $0.04 \mathrm{mg} / \mathrm{kg}$ of medetomidine was administered to obtain a persistent state that shows neuronal and BOLD activity that resembles the awake state (Schwalm et al., 2017). We confirmed the persistent brain states based on an additional scan applying visual stimuli, yielding localized activation of the primary visual cortex, in sharp contrast to the cortexwide activation in slow-wave state. Five minutes later, isoflurane anesthesia was turned off and medetomidine $0.08 \mathrm{mg} / \mathrm{kg} / \mathrm{h}$ was perfused until the end of the experiment. rsfMRI scans were performed 45, 60, 75, 90, and $120 \mathrm{~min}$ after L-DOPA/placebo administration in line with previous rodent studies showing that striatal dopamine peaks between 60 and 90 min after L-DOPA administration (Fornai et al., 1999).

fALFF analysis (humans and rats). The amplitude of low-frequency fluctuations (ALFFs) of the rsfMRI signal has been introduced to assess the intensity of regional spontaneous brain activity in humans (Zang et al., 2007). To reduce the sensitivity to physiological noise, Zou et al. (2008) developed fALFF, which is defined as the ratio of the lowfrequency amplitudes $(0.01-0.08 \mathrm{~Hz})$ to the amplitudes of the entire frequency range $(0-0.25 \mathrm{~Hz})$. In humans, fALFF analyses on preprocessed rsfMRI data were performed using the REST toolbox (Zang et al., 2007). In rats, the same frequencies were analyzed using an in-house MATLAB script (The MathWorks). Because small changes in anesthesia levels can modify dramatically the amplitude of low-frequency fluctuations in rats (Maandag et al., 2007), the obtained values were then normalized by the mean fALFF of the cortical and subcortical structures.

ROI analysis (humans). To test our a priori hypothesis of dopaminergic system activation, ROI analyses in humans were performed for a combined bilateral dopaminergic system mask (including the NAcc, caudate [Caud], putamen [Put], substantia nigra [SN], and the VTA). NAcc, Caud, and Put masks were created based on the Harvard Oxford (HO) brain atlas (Frazier et al., 2005; Desikan et al., 2006; Makris et al., 2006; Goldstein et al., 2007) using a tissue probability cutoff threshold of 50\%. The SN and the VTA were combined in a single previously published mask (Bunzeck and Düzel, 2006; Düzel et al., 2009).

In the tDCS and the L-DOPA study in humans, fALFF values of voxels within the dopaminergic system mask were averaged and analyzed in a repeated-measures ANOVA using SPSS version 23 (IBM). In the tDCS study, stimulation group (main, inv) and time (pre, post) were entered as between-subject and within-subject factors, respectively. In the L-DOPA study, both treatment and time $(0,45$, and 90 min after drug administration) were entered as within-subject factors. Partial $\eta$-squared values $\left(\eta_{p}^{2}\right)$ are reported as effect size measures. ANOVA results were further characterized by Bonferroni-corrected post hoc two-tailed $t$ tests (tDCS study: post- vs pre-tDCS in both groups; L-DOPA study: 45 vs 0 min, 90 vs $0 \mathrm{~min}$ in both conditions). Bonferroni-corrected $p$ values are denoted as $\mathrm{p}_{\text {Bonf }} \cdot \mathrm{p}_{\text {Bonf }}$ values $>1$ are reported as $\mathrm{p}_{\text {Bonf }}=1$.

A potential baseline difference between the main and the inverse group in the tDCS study and between the L-DOPA and placebo condition in the L-DOPA study was tested by two-tailed $t$ tests. Gender-related effects in the tDCS study were tested by adding gender as between-subject factor to the repeated-measures ANOVA. In the L-DOPA study, effects related to treatment order were tested by adding treatment order as betweensubject factor.

A spectral analysis of mesostriatal rsfMRI time courses was performed in the tDCS and in the human L-DOPA study using the REST toolbox (Zang et al., 2007) to inspect whether non-resting-state frequencies were affected by the two manipulations. For each subject, power spectra were calculated from unfiltered time courses of voxels within the dopaminergic system mask. Next, power spectra were averaged across voxels, smoothed by a moving average, and finally normalized.

ROI analysis (rats). A mask, covering the entire striatum and the $\mathrm{SN}$, was applied as dopaminergic system mask in the L-DOPA rat study using the atlas template from Valdés-Hernández et al. (2011). Averaged fALFF values were analyzed in a repeated-measures ANOVA with treatment and time (45, 60, 75, and 90 min after drug administration) as withinsubject factors using SPSS version 23 (IBM). Significant effects were further characterized by means of two-tailed $t$ tests (uncorrected).

Voxelwise analysis (humans). To investigate the anatomical distribution of fALFF effects within the human dopaminergic system, voxelwise analyses were performed for each subregion of the dopaminergic system mask (bilateral NAcc, Put, Caud, and the SN/VTA) using the MATLAB toolbox Statistical Parametric Mapping 8 (SPM8, Wellcome Trust Centre for Neuroimaging). fALFF values were entered into a group analysis using SPM's flexible factorial design. In the tDCS study, stimulation group and time were entered as between-subject and within-subject factors, respectively. In the L-DOPA study, both treatment and time were entered as within-subject factors. tDCS-induced activation in the main compared with the inverse group was tested by the following contrast: $\left[\mathrm{tDCS}_{\text {main,post }}-\mathrm{tDCS}_{\text {main,pre }}\right]>\left[\mathrm{tDCS}_{\text {inv,post }}-\mathrm{tDCS}_{\text {inv,pre }}\right]$. To test for L-DOPA-induced effects after 45 and $90 \mathrm{~min}$, the following contrasts were calculated: $45 \mathrm{~min},\left[\mathrm{LD}_{45 \text { min }}-\mathrm{LD}_{0 \text { min }}\right]>\left[\mathrm{Plc}_{45 \text { min }}-\mathrm{Plc}_{0 \text { min }}\right] ; 90$ $\min ,\left[\mathrm{LD}_{90 \text { min }}-\mathrm{LD}_{0 \text { min }}\right]>\left[\mathrm{Plc}_{90 \text { min }}-\mathrm{Plc}_{0 \text { min }}\right]$. Baseline differences between the tDCS groups and the L-DOPA/placebo condition were tested in all subregions of the dopaminergic system in both directions (tDCS $_{\text {main,pre }}>\mathrm{tDCS}_{\text {inv,pre }}, \mathrm{tDCS}_{\text {main,pre }}<\mathrm{tDCS}_{\text {inv,pre }} ; \mathrm{LD}_{0 \text { min }}>$ $\mathrm{Plc}_{0 \text { min }}, \mathrm{LD}_{0 \text { min }}<\mathrm{Plc}_{0 \text { min }}$ ). Family-wise error (FWE) correction was performed for voxel-level inference as implemented in SPM8 at a threshold of $\alpha=0.05$ for each region (small-volume correction [SVC]).

In both studies, the peak voxel cluster was further characterized by a functional connectivity (FC) analysis. FC maps were $z$-transformed and analyzed on a whole-brain level using SPM's flexible factorial design (see above for contrasts).

Analysis of subcortical activation profiles (humans). To examine tDCSand L-DOPA-induced effects in single subregions of the dopaminergic system (bilateral NAcc, Put, and Caud) and to test whether subcortical regions that are not part of the predefined dopaminergic system mask (bilateral hippocampus, amygdala, thalamus, pallidum, and the brainstem) were significantly affected, we analyzed changes in averaged fALFF values for the entire set of 15 subcortical gray matter $\mathrm{HO}$ atlas regions. tDCS- and L-DOPA-related activation was analyzed by means of Bonferroni-corrected two-tailed $t$ tests (tDCS, [ $\mathrm{tDCS}_{\text {main,post }}-$ $\left.\mathrm{tDCS}_{\text {main,pre }}\right]$ vs $\left[\mathrm{tDCS}_{\text {inv,post }}-\mathrm{tDCS}_{\text {inv,pre }}\right] ;$ L-DOPA (45 min), $\left[\mathrm{LD}_{45} \mathrm{~min}-\mathrm{LD}_{0 \mathrm{~min}}\right]-\left[\mathrm{Plc}_{45} \min -\mathrm{Plc}_{0}\right.$ min $]$; L-DOPA $(90 \mathrm{~min})$, $\left.\left[\mathrm{LD}_{90 \text { min }}-\mathrm{LD}_{0 \text { min }}\right]-\left[\mathrm{Plc}_{90 \text { min }}-\mathrm{Plc}_{0 \text { min }}\right]\right)$. To assess the relationship of tDCS- and L-DOPA-induced subcortical activation profiles, corre- 
sponding effect sizes (Cohen's $d$ ) were calculated for each single region and compared between the two interventions by means of Pearson correlation ( $\mathrm{r}, \alpha=0.05)$.

Analysis of gene-fALFF similarity profiles (humans). We compared uncorrected group-level statistical parametric maps ( $t$ maps) resulting from voxelwise analyses with gene expression maps based on transcriptional data from the AIB (Hawrylycz et al., 2012). Our aim was to examine similarities between brain activation maps and different neuronal receptor gene expression patterns, including those of the dopamine receptors D1-D5 (DRD1-DRD5). The AIB provides whole-brain-sampled transcriptional data of $>20,000$ genes based on six postmortem brains. Averaged and smoothed (6 mm hard sphere) AIB gene expression maps were available from the Neurosynth database (Yarkoni et al., 2011). We restricted our analysis to genes encoding receptors of 11 preselected neurotransmitters and neuromodulatory ligands, resulting in a total number of 115 genes (adrenergic receptors: ADRA1A, ADRA1B, ADRA1D, ADRA2A-C, ADRB1-3; cholinergic receptors: CHRM1-5, CHRNA1-5, CHRNA7-10, CHRNB1-4, CHRND, CHRNE, CHRNG; cannabinoid receptors: CRN1, CRN2; corticotropin releasing hormone receptors: CRHR1, CRHR2; dopamine receptors: DRD1-5; GABA receptors: GABARAP, GABBR1, GABBR2, GABARAPL1, GABARAPL2, GABRA1-6, GABRB1-3, GABRD, GABRE, GABRG1-3, GABRP, GABRQ, GABRR1-3; glutamate receptors: GRIA1-4, GRID1-2, GRIK1-5, GRIN1, GRIN2A-D, GRIN3A-B, GRINA, GRIP1-2, GRM1-8; 5-hydroxytryptamine receptors: HTR1A-B, HTR1D-F, HTR2A-C, HTR3A-E, HTR4, HTR5A, HTR6-7; glucocorticoid receptor: NR3C1; BDNF receptor: NTRK2; opioid receptors: OPRD1, OPRK1, OPRL1, OPRM1). Pearson correlations were calculated between $t$ maps ( $\mathrm{tDCS},\left[\mathrm{tDCS}_{\text {main,post }}-\mathrm{tDCS}_{\text {main,pre }}\right]>$ $\left[\mathrm{tDCS}_{\text {inv,post }}-\mathrm{tDCS}_{\text {inv,pre }}\right] ; \mathrm{L}-\mathrm{DOPA},\left[\mathrm{LD}_{45 \mathrm{~min}}-\mathrm{LD}_{0 \mathrm{~min}}\right]>\left[\mathrm{Plc}_{45 \mathrm{~min}}\right.$ $\left.\left.-\mathrm{Plc}_{0 \text { min }}\right] ; 90 \mathrm{~min},\left[\mathrm{LD}_{90 \text { min }}-\mathrm{LD}_{0 \text { min }}\right]>\left[\mathrm{Plc}_{90 \text { min }}-\mathrm{Plc}_{0 \text { min }}\right]\right)$ and gene expression maps. $t$ values of different voxels with the same gene expression intensity were averaged so that each unique gene expression intensity value was paired with a single averaged $t$ value in the correlation analysis. For each contrast, we obtained a profile of correlation coefficients, indicating similarities between the induced fALFF pattern and 115 gene expression patterns (gene-fALFF similarity profile = GFS profile). GFS profiles were $z$-transformed and compared between the two studies using Pearson correlations $(r, \alpha=0.05)$ to analyze to what extent both interventions showed comparable receptor-specific activation patterns. To furthermore assess the relative specificity for dopaminergic target regions, we determined for both interventions the percentage of genes showing higher $z$ scores than a specific dopamine receptor gene (i.e., a small percentage indicates a high specificity). The analysis was performed for all dopamine receptors (DRD1-DRD5).

\section{Data recording and processing}

rsfMRI data recording (humans). In humans, rsfMRI data were obtained with a 3 Tesla MR scanner (MAGNETOM Trio; Siemens) by using a 32-channel head coil. BOLD signal was acquired using a T2*-sensitive gradient EPI sequence with simultaneous multislice acquisition technique (TR: $1.0 \mathrm{~s}$; TE: $29 \mathrm{~ms}$; multiband acceleration factor: 4; FOV: $210 \times$ $180 \mathrm{~mm} ; 2 \times 2 \mathrm{~mm}$ in-plane resolution). Each 3D image comprised 60 contiguous axial slices ( $2.5 \mathrm{~mm}$ thick). The position of the slice package was individually adjusted for whole-brain image acquisition. Participants were instructed to remain awake with their eyes open. A fixation cross was presented on the screen center during scans. Time series of 600 rsfMRI images were acquired per session (10 $\mathrm{min})$ in the tDCS study and $480 \mathrm{rsfMRI}$ images per session ( $8 \mathrm{~min}$ ) in the L-DOPA study. To account for $\mathrm{T} 1$ equilibrium effects, the first five images of each time series were discarded. This resulted in 595 and 475 rsfMRI images per session for the tDCS and the L-DOPA study, respectively. At the end of the experiment, a high-resolution T1-weighted structural image was further acquired.

rsfMRI data preprocessing (humans). rsfMRI data were preprocessed using SPM8. Preprocessing of the rsfMRI data first involved realignment to correct for head movements. rsfMRI data were then coregistered with corresponding $\mathrm{T}_{1}$-weighted anatomical images and normalized to a standard template from the MNI to allow for group comparisons. Finally, rsfMRI data were spatially smoothed with a $6 \mathrm{~mm}$ FWHM isotropic Gaussian kernel. Participants showing head displacements $>2.5 \mathrm{~mm}$

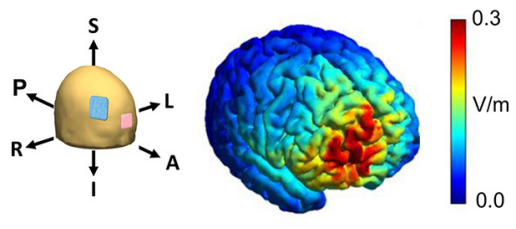

Figure 1. $\quad t D C S$ electric field simulation. Anode placement over the frontopolar/vmPFC (red represents Fpz; left) and cathode placement over the right dIPFC (blue represents F4; left) generated an electric field with maximal field strength in right lateral prefrontal areas (right).

(slice thickness; values were extracted from realignment parameters) were excluded. To further correct for possible effects of movement and other systemic effects, the six movement parameters resulting from realignment and mean CSF and white matter time series were regressed out.

Mean framewise displacements (humans). We analyzed mean framewise displacements (Power et al., 2012) to test whether tDCS and L-DOPA had specific effects on head movement and whether head movement differed between tDCS groups. Mean framewise displacements were calculated from movement parameters and analyzed in repeatedmeasures ANOVAs (tDCS: stimulation $\times$ time interaction; L-DOPA: treatment $\times$ time interaction; for a detailed description of the ANOVA design, see ROI analysis).

rsfMRI data recording and preprocessing (rats). rsfMRI data acquisition was performed on a $9.4 \mathrm{~T}$ small animal imaging system with a $0.7 \mathrm{~T} / \mathrm{m}$ gradient system (Biospec 94/20, Bruker Biospin). For rsfMRI measurements, $\mathrm{T} 2^{\star}$-weighted images were acquired with a single-shot gradient EPI sequence with $\mathrm{TR}=1.5 \mathrm{~s}$, TE $=14 \mathrm{~ms}$, FA $65^{\circ}, 320 \times 290 \mu \mathrm{m}$, slice thickness $0.8 \mathrm{~mm}$. Together, 600 images, each comprising 34 contiguous slices, were acquired per scan, resulting in a scan time of $15 \mathrm{~min}$. Each scan of 600 images was preprocessed using Brain Voyager QX (version 2.8.4, Brain Innovation). rsfMRI data were realigned to correct for head movements and smoothed with a $0.7 \mathrm{~mm}$ FWHM isotropic Gaussian kernel. Using the same software, rsfMRI data were manually aligned to a T1 template from (Valdés-Hernández et al., 2011) containing 150 cortical and subcortical regions. Finally, a high-resolution T1-weighted structural image $(0.125 \times 0.125 \times 0.125 \mathrm{~mm})$ was acquired.

\section{Results}

Side effect reports and mean framewise displacements

All participants of the tDCS study reported a mild tingling under the electrodes during stimulation, regardless of the group. However, no other somatic or psychological side effects that were attributable to the intervention were reported. Likewise, no treatment-related side effects were reported in the L-DOPA study. Analyzing mean framewise displacements revealed that tDCS and L-DOPA had no specific effects on head movement (tDCS: stimulation $\times$ time interaction: $F_{(1,38)}=1.28, p=0.264$; L-DOPA study: treatment $\times$ time interaction: $F_{(2,42)}=0.7, p=$ $0.503)$, and there was also no significant difference between tDCS groups $\left(F_{(1,38)}=0.65, p=0.426\right)$.

\section{Electric field simulation (tDCS study)}

A simulation of the electric field (anode: Fpz, cathode: F4) indicated maximal electric field strength $(\sim 0.3 \mathrm{~V} / \mathrm{m})$ in the right superior and middle frontal gyrus (Fig. 1). Field strengths were comparable with those generated by other prefrontal montages (see, e.g., Saturnino et al., 2015), suggesting that a substantial amount of current passed through cortical areas.

\section{ROI analysis (tDCS study)}

We first tested our a priori hypothesis that stimulation in the main group remotely activates brain areas of the subcortical dopaminergic system compared with stimulation with inverse polarity. When averaging across voxels in a dopaminergic sys- 
tem mask (Fig. 2A), we found significant main effects of time $\left(F_{(1,38)}=22.16\right.$, $\left.p<0.001, \eta_{p}^{2}=0.37\right)$ and stimulation $\left(F_{(1,38)}=7.3, p=0.01, \eta_{p}^{2}=0.16\right)$, which however could be explained by a significant stimulation $\times$ time interaction $\left(F_{(1,38)}=13.77, p<0.001, \eta_{p}^{2}=0.266\right.$; Fig. $2 B$ ). A strong fALFF increase in the main as opposed to the inverse tDCS group from prestimulation to poststimulation was supported by post hoc $t$ tests (main: $t_{(19)}=5.08, \mathrm{p}_{\text {Bonf }}<0.001$; inv: $t_{(19)}$ $\left.=0.89, \mathrm{p}_{\text {Bonf }}=0.77\right)$. Power spectra, calculated from time courses of masked mesostriatal voxels, indicated that tDCS most strongly amplified classical restingstate frequencies ranging from 0.01 to $0.08 \mathrm{~Hz}$ (Fig. 3A).

There was no significant fALFF difference between the two groups at baseline $\left.t_{(38)}=-1.22, p=0.231\right)$, and no significant effects of gender were found (main effect of gender: $F_{(1,36)}=0.68, p=0.416$; time $\times$ stimulation $X$ gender interaction: $\left.F_{(1,36)}=0.22, p=0.642\right)$.

\section{ROI analysis (L-DOPA study in humans)}

We found a significant treatment $\times$ time interaction indicating an L-DOPAinduced fALFF increase in the dopaminergic system $\left(F_{(2,42)}=4.17, p=0.022\right.$, $\eta_{p}^{2}=0.166$; Fig. $\left.2 C\right)$. The interaction subsumed a main effect of time $\left(F_{(2,42)}=\right.$ $\left.5.34, p=0.009, \eta_{p}^{2}=0.20\right)$. Post hoc $t$ tests revealed a significant fALFF increase 90 min, but not $45 \mathrm{~min}$, after L-DOPA administration (90 min: $t_{(21)}=4.35, \mathrm{p}_{\mathrm{Bonf}}<$ $\left.0.001 ; 45 \mathrm{~min}: t_{(21)}=1.17, \mathrm{p}_{\text {Bonf }}=1\right)$. fALFF increases after placebo administration were not supported $\left(90 \mathrm{~min}: t_{(21)}=\right.$ $0.66, \mathrm{p}_{\text {Bonf }}=1 ; 45 \mathrm{~min}: t_{(21)}=0.48, \mathrm{p}_{\text {Bonf }}=$ $1)$. As for $\mathrm{tDCS}$, the spectral power analysis supported major effects on resting-state frequencies (Fig. 3B).

Treatment order had no significant effect on fALFF values (main effect: $F_{(1,20)}=$ $0.02, p=0.878$; treatment $\times$ time $\times$ order interaction: $\left.F_{(2,40)}=1.96, p=0.154\right)$. There was no significant difference between the two conditions at baseline $\left(t_{(21)}=0.95\right.$, $p=0.354)$.

\section{ROI analysis (L-DOPA study in rats)}

In an additional L-DOPA study, we conducted a comparable experimental approach in rats, masking the homologous brain regions, with recordings performed at 45, 60, 75, 90, and 120 min after LDOPA/placebo administration. As in the human studies, we performed an ROI analysis on averaged fALFF values and found that L-DOPA led to a significant fALFF increase that reached its peak after 45-75 min (treatment $\times$ time interaction: $F_{(4,20)}=3.62, p=$ $0.023, \eta_{p}^{2}=0.43$; Fig. 4). $\left({ }^{* *} p<0.01 ;{ }^{* * *} p<0.001\right)$.
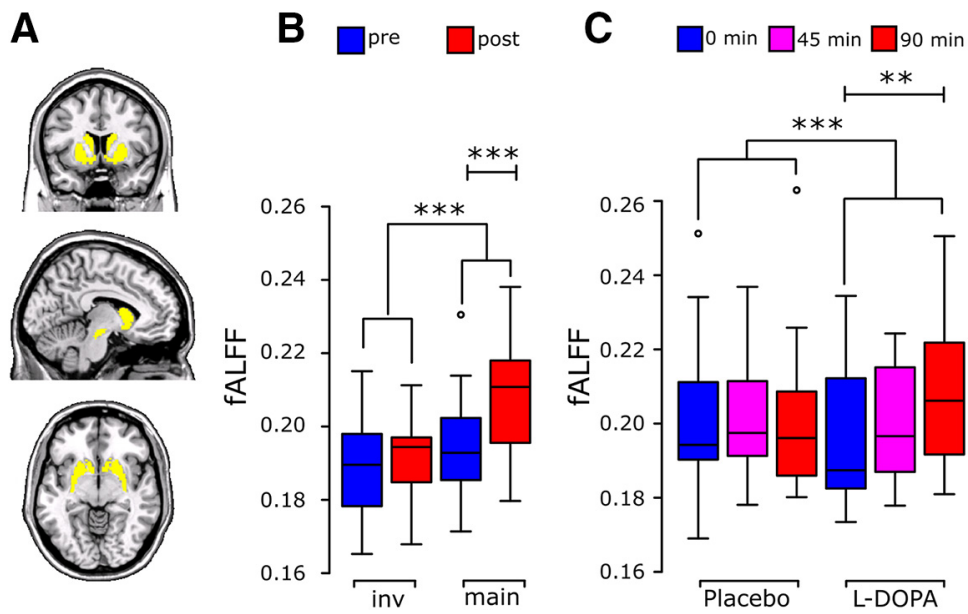

Figure 2. $\quad$ tDCS- and L-DOPA-induced effects in the mesostriatal system (ROI analysis). Using a human dopaminergic system mask $(\boldsymbol{A})$, averaged fALFF increases were observed after tDCS in the main but not in the inverse group $(\boldsymbol{B})$ and 90 min after L-DOPA administration (C). Boxplots represent median, quartiles (boxes), and range (whiskers). Whiskers extend 1.5 times the interquartile range. Dots represent outliers. Asterisks indicate levels of statistical significance for interaction contrasts (tDCS: $\left[\mathrm{tDCS}_{\text {main,post }}-\right.$ $\mathrm{tDCS}$ main,pre $] v s\left[\mathrm{tDCS}\right.$ inv, post $\left.\left.-\mathrm{tDCS} \mathrm{inv}_{\text {, pre }}\right] ; \mathrm{L}-\mathrm{DOPA}:\left[\mathrm{LD}_{90 \text { min }}-\mathrm{LD}_{0 \text { min }}\right]-\left[\mathrm{Pl}_{90 \text { min }}-\mathrm{Pl}_{0 \text { min }}\right]\right)$ and Bonferroni-corrected post hoct tests
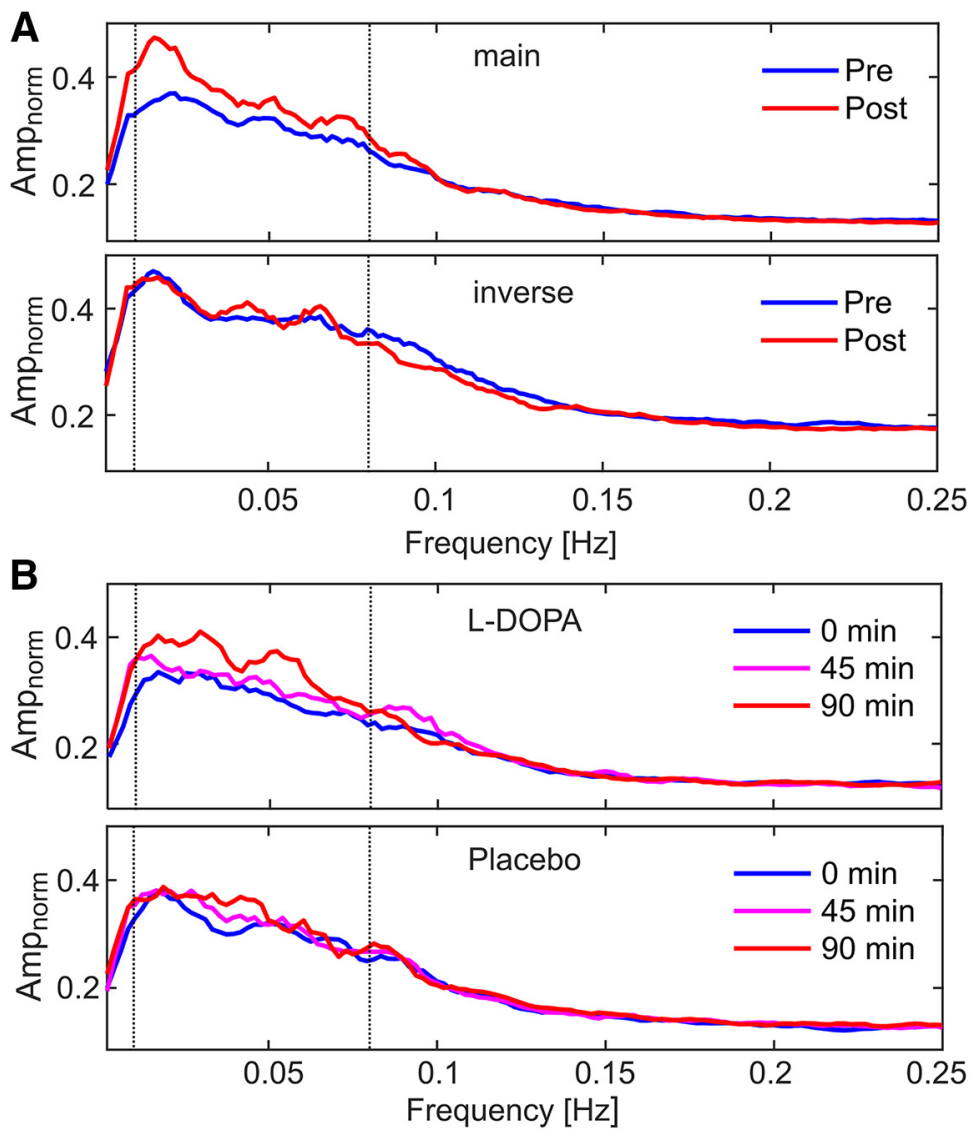

Figure 3. Spectral analyses of the fMRI signal in the dopaminergic system revealed that both $\operatorname{tDCS}(\boldsymbol{A})$ and L-DOPA $(\boldsymbol{B})$ amplified characteristic resting-state frequencies. Dashed lines indicate $0.01-0.08 \mathrm{~Hz}$.

\section{Voxelwise analysis (tDCS study)}

Examining the voxelwise spatial distribution of tDCS-induced effects within subregions of the dopaminergic system revealed significant activations in multiple striatal areas, including the bilateral Caud, NAcc, Put, and the SN/VTA (Table 1). The right Put showed the strongest activation cluster, which was mainly located in dorsal parts of the striatum (Fig. 5). None of 


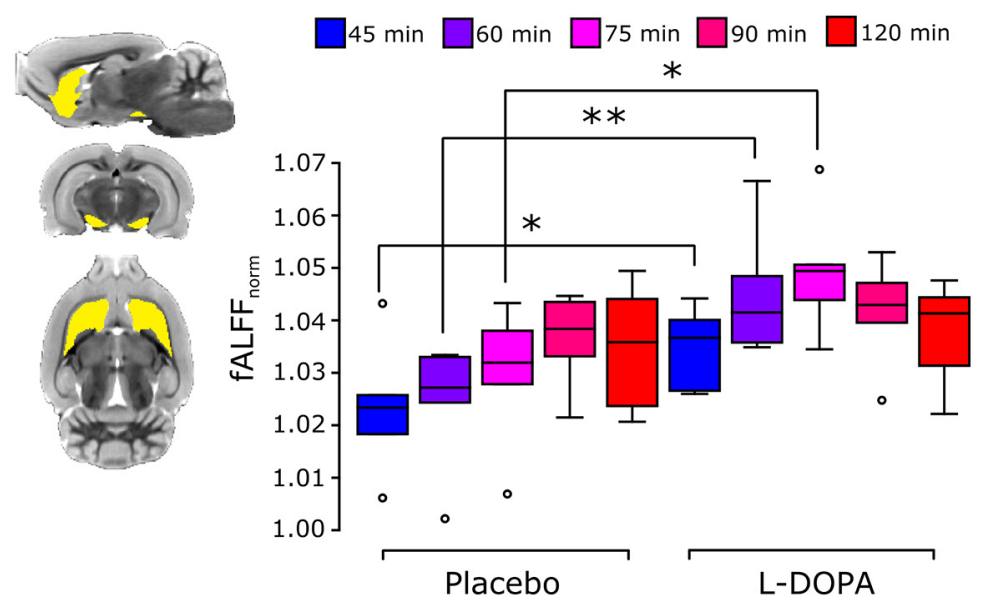

Figure 4. L-DOPA-induced effects in the mesostriatal system of rats (ROI analysis). In rats, we found L-DOPA-induced fALFF increases after 45, 60, and 75 min when averaging across voxels in a dopaminergic system mask. Box plots represent median, quartiles (boxes), and range (whiskers). Whiskers extend 1.5 times the interquartile range. Dots represent outliers. Statistical significance for uncorrected post hoc $t$ tests: ${ }^{*} p<0.05 ;{ }^{* *} p<0.01$.

Table 1. tDCS-induced effects in the mesostriatal system (voxelwise analysis) ${ }^{a}$

\begin{tabular}{llllll}
\hline ROI & MNI $(\mathrm{mm})$ & Cluster size & $t$ & $z$ & $p_{\mathrm{FWE}}{ }^{b}$ \\
\hline Right putamen & $32,-8,0$ & 286 & 5.25 & 4.52 & 0.002 \\
Left caudate & $-14,16,4$ & 58 & 4.18 & 3.77 & 0.026 \\
Left putamen & $-26,2,0$ & 192 & 4.26 & 3.83 & 0.035 \\
Right caudate & $12,10,10$ & 15 & 3.98 & 3.61 & 0.043 \\
SN/VTA & $-10,-22,-12$ & 7 & 3.59 & 3.31 & 0.045
\end{tabular}

${ }^{a}$ Peak voxels indicating $\mathrm{DCC}$-induced neural activity in the main as opposed to the inverse group in mesostriatal subregions. Coordinates are denoted by $x, y, z$ (MNI space).

${ }^{b}$ Small-volume FWE-corrected $p$ values for voxel-level inference.

the mesostriatal regions showed a baseline difference between the groups.

Extending the analysis of tDCS-induced effects to other subcortical HO atlas regions (bilateral hippocampus, amygdala, thalamus, pallidum, and the brainstem) showed no further significant results, and no significant FC changes were found for the right Put (peak voxel cluster).

\section{Voxelwise analysis (L-DOPA study in humans)}

In contrast to the analysis of averaged fALFF values, voxelwise analyses revealed a significant activation $45 \mathrm{~min}$ after L-DOPA administration that was restricted to the SN/VTA. After $90 \mathrm{~min}$, L-DOPA-induced effects were found in ventral parts of the striatum, where the strongest activation was observed at the borderline between the left NAcc, Caud, and Put (Table 2; Fig. 5). None of the mesostriatal regions showed a significant baseline difference.

An exploratory analysis of all subcortical $\mathrm{HO}$ atlas regions furthermore indicated L-DOPA-induced activations after $90 \mathrm{~min}$ in the brainstem $\left(x, y, z: 6,-38,-44 ; \mathrm{p}_{\mathrm{FWE}}=0.009\right.$, SVC), left pallidum $\left(x, y, z:-24,-12,0 ; \mathrm{p}_{\mathrm{FWE}}=0.028\right.$, SVC), left thalamus $\left(x, y, z:-12,-28,12 ; \mathrm{p}_{\mathrm{FWE}}=0.027, \mathrm{SVC}\right)$ and the left amygdala $\left(x, y, z:-30,6,-22 ; \mathrm{p}_{\mathrm{FWE}}=0.049, \mathrm{SVC}\right)$. No further activations were found when analyzing the time window 45 min after treatment administration. The ventral striatum peak cluster showed a significant L-DOPA-induced FC increase with the right cerebellum after $90 \min \left(x, y, z: 38,-52,-32\right.$; $\mathrm{p}_{\mathrm{FWE}}=0.026$, wholebrain analysis). No significant L-DOPA-induced FC changes were found for the SN/VTA in the time window 45 min after treatment administration.

\section{Analysis of subcortical activation profiles}

We examined tDCS-induced activity changes (for each subcortical $\mathrm{HO}$ atlas region) on a regionwise anatomical scale and again found peak activations in key regions of the mesostriatal dopamine system. The right and left Put and Caud constituted the four most activated subcortical brain regions, of which the Put on both sides reached the level of Bonferronicorrected statistical significance ( $\left[\mathrm{tDCS}_{\text {main,post }}-\mathrm{tDCS}_{\text {main,pre }}\right]$ vs [tDC$\left.\mathrm{S}_{\text {inv,post }}-\mathrm{tDCS}_{\text {inv,pre }}\right]$; right Put: $t_{(38)}=$ 3.93, p $_{\text {Bonf }}=0.005$; left Put: $t_{(38)}=3.17$, $\left.\mathrm{p}_{\text {Bonf }}=0.045\right)$. In the L-DOPA study, the right and left Caud constituted the most activated subcortical brain regions 90 min after L-DOPA intake, of which the latter reached the level of Bonferronicorrected statistical significance $\left(\left[\mathrm{LD}_{90} \mathrm{~min}\right.\right.$ $\left.-\mathrm{LD}_{0 \text { min }}\right]-\left[\mathrm{Plc}_{90 \text { min }}-\mathrm{Plc}_{0 \text { min }}\right] ; t_{(21)}=$ 3.37, $\left.\mathrm{p}_{\mathrm{Bonf}}=0.045\right)$. No significant activation was found $45 \mathrm{~min}$ after L-DOPA administration.

To compare subcortical activation profiles of both interventions, we next calculated effect sizes (Cohen's $d$ ) for all subcortical $\mathrm{HO}$ atlas regions. No significant relationship was found when comparing the effect size profiles for L-DOPA after $45 \mathrm{~min}$ with tDCS $(r=-0.19, p=0.504)$. However, there was a significant correlation when comparing the profiles for L-DOPA after 90 min and tDCS ( $r=0.73, p=0.002$; Fig. 6$)$, suggesting a potential analogy of tDCS- and L-DOPA-induced subcortical effects.

\section{Analysis of gene-fALFF similarity profiles}

We finally calculated correlations between tDCS-/L-DOPArelated brain activation maps (uncorrected whole-brain $t$ maps resulting from voxelwise analyses) and 115 receptor gene expression patterns (GFS profiles) to examine the relative specificity of both interventions for regions with high dopamine receptor expression and to compare GFS profiles between the two interventions. $z$-transformed GFS profiles showed a strong correlation indicating that tDCS and L-DOPA activated brain regions with similar receptor composition (tDCS vs L-DOPA (90 min): $r=$ $0.88, p<0.001$; tDCS vs L-DOPA $(45 \mathrm{~min}): r=0.82, p<0.001$; Figure 7).

Among all 115 preselected receptor genes, the DRD2 expression pattern most strongly resembled the activation pattern induced by tDCS, and only $1.74 \%$ of the gene expression maps (i.e., two gene expression maps) correlated more with the tDCS-induced activation pattern than the DRD3 expression map ( $D R D 2$ and the $\varepsilon$ subunit of the GABA A receptor [GABRE]). DRD2 and DRD3 expression maps also showed a strong correlation with the L-DOPA-induced pattern after 90 min compared with other genes: As for tDCS, no other gene expression map showed a stronger correlation than DRD2, and only four gene expression maps $(3.48 \%)$ correlated more with the L-DOPA-induced pattern than DRD3 (DRD2, 5-hydroxytryptamine receptor 1D $[H T R 1 D], \alpha-2 \mathrm{~B}$ adrenergic receptor $[A D R A 2 B], \alpha-2 C$ adrenergic receptor $[A D R A 2 C]$; Fig. 7). Hence, both interventions showed a high similarity in their receptor-specific activation patterns and specifically activated regions with high $D R D 2$ and $D R D 3$ expression levels.

Analyzing the L-DOPA effect 45 min after administration revealed that $5.21 \%$ and $7.83 \%$ of all gene expression maps showed 

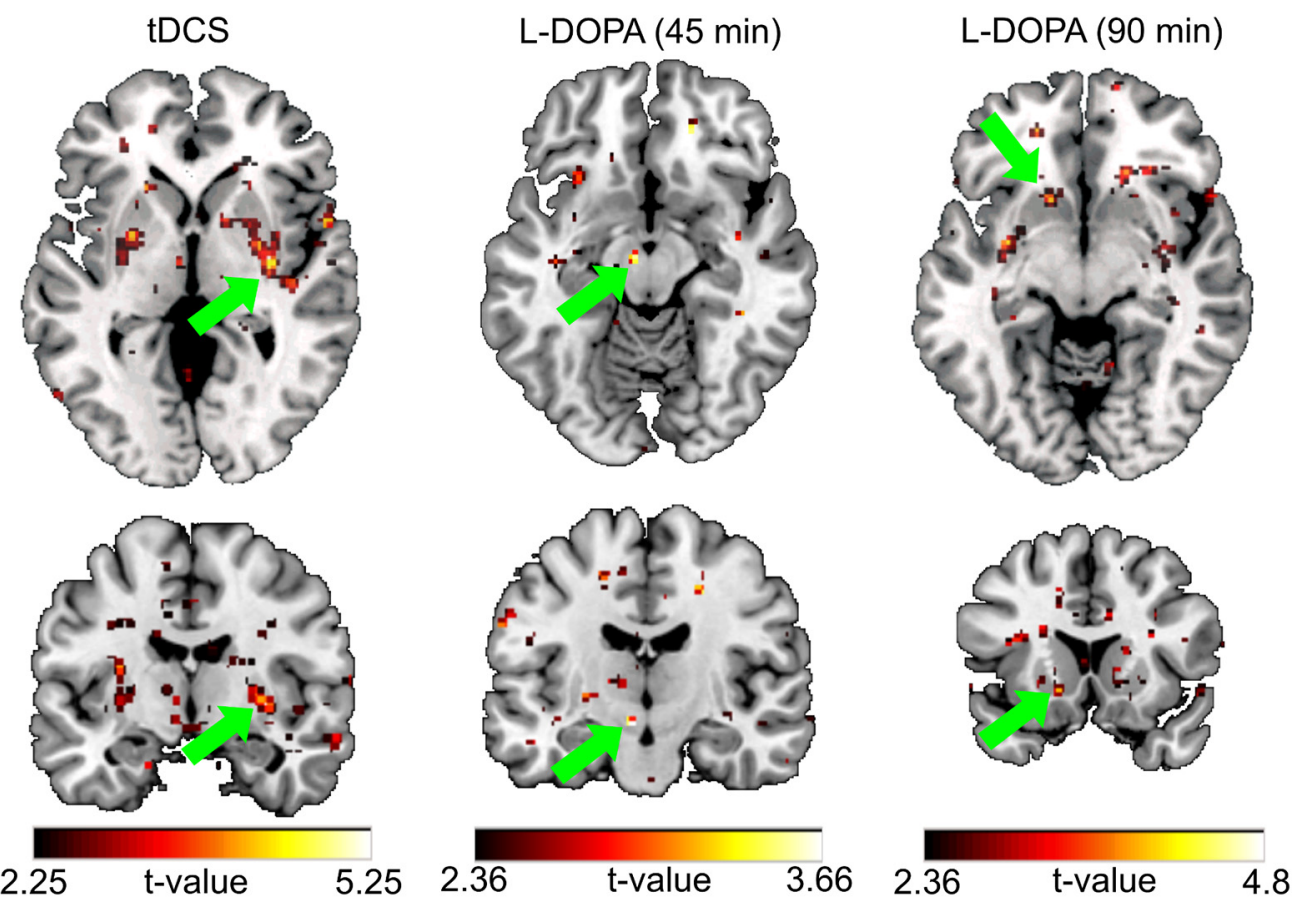

Figure 5. $\quad$ tDCS- and L-DOPA-induced effects in the mesostriatal system (voxelwise analysis). tDCS-induced activation in the main compared with the inverse group was restricted to areas of the dopaminergic system and most pronounced in the right putamen. L-DOPA compared with placebo administration led to a significant activation of the SN/VTA after 45 min and of the left ventral striatum after $90 \mathrm{~min}$. Green arrows indicate peak voxels. Small-volume FWE correction was performed for voxel-level inference. The display threshold was set to $p<0.01$ (uncorrected).

Table 2. L-DOPA-induced effects in the mesostriatal system (voxelwise analysis) ${ }^{a}$

\begin{tabular}{|c|c|c|c|c|c|}
\hline $\mathrm{ROI}$ & MNI (mm) & Cluster size & $t$ & $z$ & $p_{\mathrm{FWE}}^{b}$ \\
\hline \multicolumn{6}{|c|}{ L-DOPA-induced activation after $45 \mathrm{~min}$} \\
\hline \multirow{3}{*}{ SN/VTA } & $-6,-14,-12$ & 12 & 3.79 & 3.66 & 0.013 \\
\hline & $-8,-24,-18$ & 16 & 3.76 & 3.64 & 0.015 \\
\hline & $6,-22,-20$ & 10 & 3.67 & 3.55 & 0.019 \\
\hline \multicolumn{6}{|c|}{ L-DOPA-induced activation after $90 \mathrm{~min}$} \\
\hline Left NAcc/caudate & $-14,16,-8$ & 11 & 3.91 & 3.77 & 0.004 \\
\hline Right NAcc/caudate & $14,18,-6$ & 3 & 3.41 & 3.32 & 0.014 \\
\hline Left putamen & $-28,-6,10$ & 55 & 4.03 & 3.88 & 0.025 \\
\hline
\end{tabular}

${ }^{a}$ Peak voxels indicating enhanced neural activity after I-DOPA as opposed to placebo administration in mesostriatal subregions after 45 and $90 \mathrm{~min}$. Coordinates are denoted by $x_{1} y, z$ (MNI space).

${ }^{b}$ Small-volume FWE-corrected $p$ values for voxel-level inference.

a stronger correlation with the brain activation pattern than $D R D 2$ and $D R D 3$, respectively. In contrast to $D R D 2$ and $D R D 3$, other dopamine receptor expression maps showed less similarity with tDCS- and L-DOPA-induced patterns, that is, more genes showed stronger correlations (tDCS: DRD1 = 14.78\%, DRD4 = 75.65\%, DRD5 = 33.04\%; L-DOPA (45 min): DRD1 $=40.87 \%$, DRD4 $=60.87 \%$, DRD5 $=51.3 \%$; L-DOPA $(90 \mathrm{~min}):$ DRD1 $=$ $8.7 \%, D R D 4=49.57 \%$, DRD5 $=57.39 \%)$. The difference between DRD2-3 on the one hand and DRD1 and DRD4-5 on the other was also reflected when analyzing correlations between the gene expression maps of the dopamine receptors indicating the strongest correlation between DRD2 and DRD3 $(r=0.67$; Table 3$)$.

\section{Discussion}

We tested the influence of prefrontal tDCS and L-DOPA on deep regions of the mesostriatal dopamine system at rest in healthy humans. Regional neural activity strength was assessed by means of fALFF analyses. Anodal/cathodal Fpz/F4 stimulation led to enhanced fALFF values in key regions of the dopaminergic system. No effects were observed when the same montage was used with inverse polarity. Increased activity in mesostriatal regions was also found after L-DOPA administration. Both interventions showed distinct similarities in subcortical and receptor-specific activation profiles, suggesting mechanistic commonalities and a potential application of prefrontal tDCS in the treatment of dopamine dysfunctions.

To achieve better comparability between studies in animals and humans, we investigated fALFF in task-free resting states. fALFF has been applied as a proxy measure of spontaneous neural activity strength and has been used increasingly as a biomarker of neuropsychiatric diseases, including Alzheimer's disease (Zhou et al., 2015), schizophrenia (Xu et al., 2015), and depression (Liu et al., 2016). Several studies reported significant correlations of fALFF values and symptom severity in patients (Chen et al., 2015; Fryer et al., 2015) as well as behavioral performance in healthy humans (van Dam et al., 2015), suggesting a close association between resting-state fALFFs and specific behavioral parameters.

We used a stimulation protocol developed by Chib et al. (2013) to remotely activate reward-related regions of the dopaminergic system. Chib et al. (2013) found increased task-related fMRI signal interactions between the vmPFC and the ventral midbrain after tDCS; and most importantly, participants with more enhanced frontomidbrain interactions assigned higher attractiveness ratings when judging the attractiveness of computer-generated faces. These results indicated, for the first time, a tDCS-induced effect on midbrain parts of the dopaminergic system with direct behavioral consequences. However, an interpretation of the results of Chib et al. (2013) from a translational perspective is not straightforward, mainly because the authors investigated the effect of $\mathrm{tDCS}$ on reward-related brain activity in a task, which is only suited for human subjects.

Considering the neuroanatomical literature, anodal stimulation of glutamatergic projections from the vmPFC to the mesostriatal system may have evoked the observed fALFF increases (see, e.g., Hedreen and DeLong, 1991; Frankle et al., 2006; Haber and 


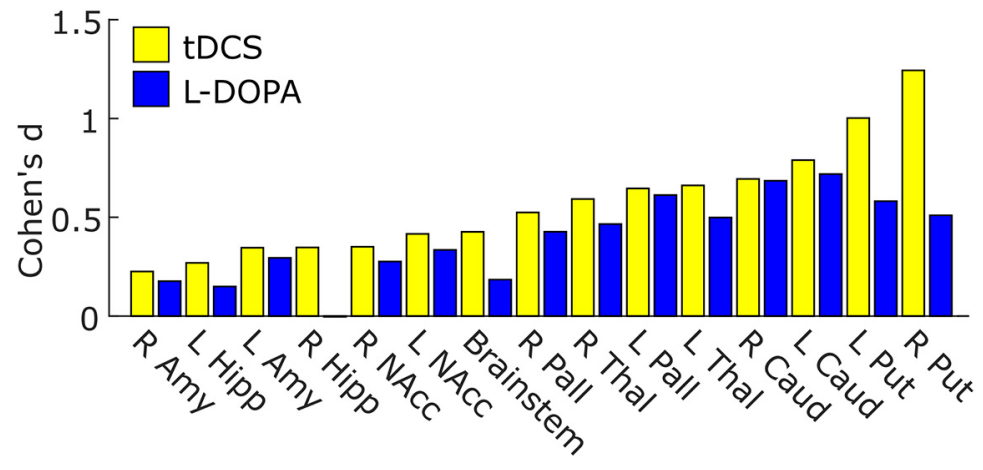

Figure 6. Subcortical activation profiles. TDCS and L-DOPA evoked similar subcortical activation profiles as revealed by a significant Pearson correlation of regionwise effect sizes (Cohen's $d$ ). Yellow represents tDCS-induced effect. Blue represents L-DOPAinduced effect after 90 minutes. $r=0.73, p=0.002$. Labels were taken from the HO atlas. R, Right; L, left; Hipp, hippocampus; Amy, amygdala; Pall, pallidum; Thal, thalamus; Caud, caudate; Put, putamen.

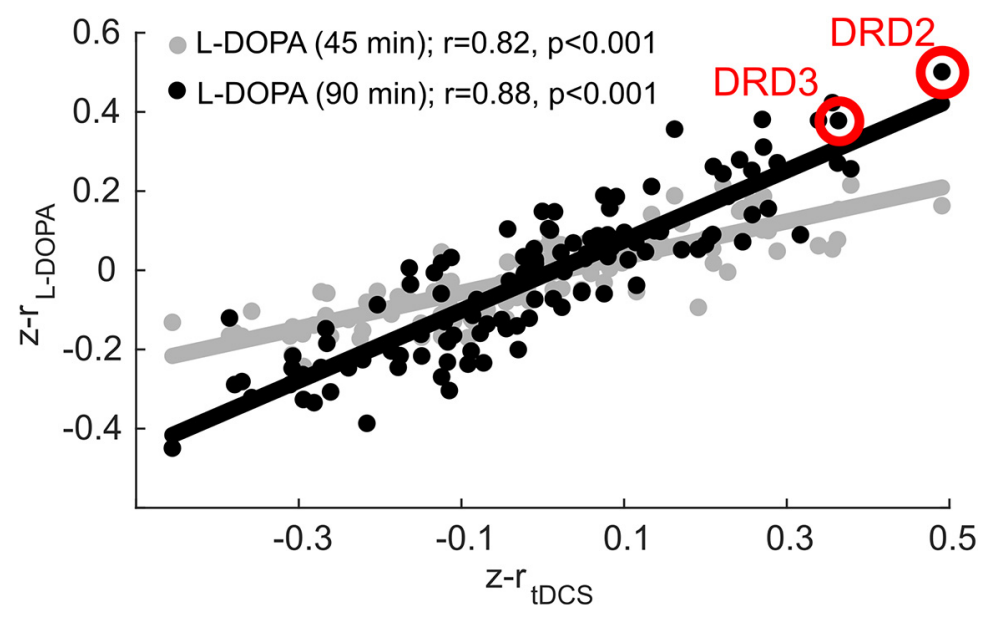

Figure 7. Gene-fALFF similarity profiles. Each point indicates the $z$-transformed spatial correlation (similarity) of a particular gene expression map and the evoked activity pattern after tDCS ( $x$ axis) and L-DOPA ( $y$ axis). Gray points indicate L-DOPA after 45 min. Black points indicate L-DOPA after $90 \mathrm{~min}$. Activity patterns induced by tDCS and L-DOPA after $90 \mathrm{~min}$ showed a pronounced similarity with gene expression patterns of the dopamine receptors D2 and D3 (DRD2, DRD3; red circles) compared with other neuronal receptor genes. There was a significant linear relationship between gene-fALFF similarity values of tDCS and L-DOPA. Gray line indicates L-DOPA after $45 \mathrm{~min}(r=0.82)$. Black line indicates L-DOPA after $90 \mathrm{~min}(r=0.88)$, indicating analogous receptorspecific activation patterns for both interventions.

Table 3. Comparison of dopamine receptor gene expression maps ${ }^{a}$

\begin{tabular}{lcccll}
\hline & DRD1 & DRD2 & DRD3 & DRD4 & DRD5 \\
\hline DRD1 & - & 0.375 & 0.49 & -0.07 & 0.39 \\
DRD2 & 0.375 & - & 0.67 & -0.06 & 0.1 \\
DRD3 & 0.49 & 0.67 & - & -0.16 & 0.04 \\
DRD4 & -0.07 & -0.06 & -0.16 & - & 0 \\
DRD5 & 0.39 & 0.1 & 0.04 & 0 & -
\end{tabular}

a Pearson correlation coefficients $(r)$ resulting from pairwise correlations of dopamine receptor gene expression maps (DRD1-DRD5).

Knutson, 2010). However, the electric field simulation indicated maximal field strength in right lateral cortical regions, furthermore suggesting a crucial role of inhibitory cathodal stimulation over the right dlPFC (F4) in the remote activation of mesostriatal regions. Interestingly, Chib et al. (2013) tested multiple electrode montages but only observed effects on dopamine-dependent functioning when placing the cathode at F4 and the anode at Fpz. Furthermore, Fonteneau et al. (2018) reported elevated striatal dopamine levels after cathodal F4 and anodal F3 stimulation. Hence, one could speculate that the right dlPFC exerts inhibitory control over the dopaminergic system, which can be deactivated through cathodal stimulation. However, overall the electric fields generated by anodal/cathodal Fpz/F4 stimulation (used here and by Chib et al., 2013) and anodal/ cathodal F3/F4 stimulation (used by Fonteneau et al., 2018) differ, which is against the hypothesis that both configurations activate the same neural pathway (for electric field maps of anodal/cathodal F3/F4 stimulation, see, e.g., Austin et al., 2016; Bikson et al., 2018). An alternative explanation for striatal effects after both anodal/cathodal Fpz/F4 and anodal/cathodal F3/F4 stimulation is provided by FC analyses in humans, showing a topological relationship between distinct cortical networks and specific striatal subregions (Choi et al., 2012). Hence, two different tDCS protocols, modulating activity in different parts of the PFC (and thus possibly in different cortical networks), may both affect the striatum but through different corticostriatal pathways and targeting different striatal subregions.

When comparing our results with those of Fonteneau et al. (2018), it should also be mentioned that Fonteneau et al. (2018) reported significant changes in dopamine release 20-35 min after tDCS but not directly after stimulation, as shown for fALFF in the present study. To fully investigate this difference, it will be required to directly compare acute and late mesostriatal effects between the two tDCS protocols using both PET and fMRI.

L-DOPA is converted to dopamine in the intracellular space of dopaminergic neurons, which form the neuroanatomical basis of dopamine-dependent neuromodulation (Volkow et al., 1996), while as previously stated, the effect of prefrontal tDCS is likely mediated by corticosubcortical projections. Both interventions induced similar subcortical activation profiles on a regionwise anatomical scale; however, voxelwise fALFF analyses also revealed intraregional differences. This is not necessarily contradictory because ascending dopaminergic projections (activated by L-DOPA) and descending cortical projections (activated by tDCS) may target different parts of the same anatomical region. From a clinical perspective, this indeed constitutes a promising finding, as it points to a complementary and more effective stimulation of the dopaminergic system by combining both interventions in the treatment of dopamine dysfunctions.

So far, there are only limited insights into the effects of L-DOPA on resting-state fALFFs as most studies primarily focused on changes in FC (see, e.g., Cole et al., 2013). One exception is the study by Flodin et al. (2012), who investigated L-DOPArelated fALFF changes in a set of cortical and subcortical ROIs, but did not find significant striatal effects. However, it should be mentioned that the authors used a between-subjects design and acquired data at only 1.5 Tesla, which might have reduced the sensitivity to detect fALFF changes. 
Using transcriptional data from the AIB, we found that both interventions manipulated neural activity in dopaminergic target regions characterized by high $D R D 2$ and $D R D 3$ expression. Although the analysis of gene expression maps may partly bridge the gap between hemodynamic effects and molecular mechanisms, it is important to note that we do not provide direct evidence for tDCS/L-DOPA-induced dopaminergic neurotransmission, which can only be achieved with molecular imaging techniques. In particular, the combination of fMRI and PET will finally help to understand the relationship between fALFF increases and dopamine release.

In an additional study, we analyzed the effects of L-DOPA in lightly sedated rats, which resembled those in awake humans. fALFF increases followed different temporal characteristics compared with humans, which however might be explained by several factors that are difficult to control, most importantly differences in administration routes, dosage, and metabolic rate. Recent rodent studies (Cha et al., 2016; Yan et al., 2017) as well as our own results support fALFF as a suitable marker of intrinsic brain activity changes in animals. Furthermore, it supports the notion that a direct (back-)translation of a network manipulation yields homologous oscillatory activity, provided that the brain state of the animal is being carefully controlled (Schwalm et al., 2017).

Several methodological drawbacks of the present approach should be mentioned. In the tDCS study, both genders were tested, whereas either only male humans or female rats were tested in the L-DOPA studies. A careful control would have been conducive to the comparability of both interventions and might have given additional information. Although experimenter effects are unlikely to occur in task-free study designs, it should be mentioned that the tDCS study was only single-blinded. In both studies, blinding efficacy was not assessed, but none of the participants reported unexpected side effects or perceived symptoms (apart from cutaneous sensations at the site of stimulation in both $\mathrm{tDCS}$ groups), which at least reduces the risk of a significant experimental bias. Finally, our tDCS study does not include a sham group to exclude unspecific time-dependent effects. However, we assume that such effects only play a minor role as they were not observed in the inverse group of the tDCS study and in the placebo condition of the L-DOPA study.

In conclusion, in line with animal studies and recent molecular imaging studies in humans, we found spatially specific tDCSrelated activity increases in subcortical parts of the dopaminergic system, particularly in the striatum. tDCS and L-DOPA showed comparable subcortical and receptor-specific activation profiles, suggesting mechanistic commonalities between both manipulations. These results are promising in respect of restoring depleted dopamine levels and may expand the repertoire of tDCS protocols that have been successfully applied in therapeutic contexts (Brunoni et al., 2012; Kalu et al., 2012). However, future studies, testing whether the approach presented here affects clinically relevant neurochemical and, most importantly, behavioral parameters are required to finally draw conclusions on its potential clinical value.

\section{References}

Appleby BS, Duggan PS, Regenberg A, Rabins PV (2007) Psychiatric and neuropsychiatric adverse events associated with deep brain stimulation: a meta-analysis of ten years' experience. Mov Disord 22:1722-1728.

Austin A, Jiga-Boy GM, Rea S, Newstead SA, Roderick S, Davis NJ, Clement RM, Boy F (2016) Prefrontal electrical stimulation in non-depressed reduces levels of reported negative affects from daily stressors. Front Psychol 7:315.

Benetello P, Furlanut M, Fortunato M, Pea F, Baraldo M (1997) Levodopa and 3-O-methyldopa in cerebrospinal fluid after levodopa-carbidopa association. Pharmacol Res 35:313-315.

Bikson M, Brunoni AR, Charvet LE, Clark VP, Cohen LG, Deng ZD, Dmochowski J, Edwards DJ, Frohlich F, Kappenman ES, Lim KO, Loo C, Mantovani A, McMullen DP, Parra LC, Pearson M, Richardson JD, Rumsey JM, Sehatpour P, Sommers D, et al. (2018) Rigor and reproducibility in research with transcranial electrical stimulation: an NIMH-sponsored workshop. Brain Stimul 11:465-480.

Brunoni AR, Nitsche MA, Bolognini N, Bikson M, Wagner T, Merabet L, Edwards DJ, Valero-Cabre A, Rotenberg A, Pascual-Leone A, Ferrucci R, Priori A, Boggio PS, Fregni F (2012) Clinical research with transcranial direct current stimulation (tDCS): challenges and future directions. Brain Stimul 5:175-195.

Bunzeck N, Düzel E (2006) Absolute coding of stimulus novelty in the human substantia nigra/VTA. Neuron 51:369-379.

Cha J, Kim ST, Jung WB, Han YH, Im GH, Lee JH (2016) Altered white matter integrity and functional connectivity of hyperacute-stage cerebral ischemia in a rat model. Magn Reson Imaging 34:1189-1198.

Chen YC, Xia W, Luo B, Muthaiah VP, Xiong Z, Zhang J, Wang J, Salvi R, Teng GJ (2015) Frequency-specific alternations in the amplitude of lowfrequency fluctuations in chronic tinnitus. Front Neural Circuits 9:67.

Chib VS, Yun K, Takahashi H, Shimojo S (2013) Noninvasive remote activation of the ventral midbrain by transcranial direct current stimulation of prefrontal cortex. Transl Psychiatry 3:e268.

Choi EY, Yeo BT, Buckner RL (2012) The organization of the human striatum estimated by intrinsic functional connectivity. J Neurophysiol 108: 2242-2263.

Cole DM, Oei NY, Soeter RP, Both S, van Gerven JM, Rombouts SA, Beckmann CF (2013) Dopamine-dependent architecture of corticosubcortical network connectivity. Cereb Cortex 23:1509-1516.

Damoiseaux JS, Rombouts SA, Barkhof F, Scheltens P, Stam CJ, Smith SM, Beckmann CF (2006) Consistent resting-state networks across healthy subjects. Proc Natl Acad Sci U S A 103:13848-13853.

Desikan RS, Ségonne F, Fischl B, Quinn BT, Dickerson BC, Blacker D, Buckner RL, Dale AM, Maguire RP, Hyman BT, Albert MS, Killiany RJ (2006) An automated labeling system for subdividing the human cerebral cortex on MRI scans into gyral based regions of interest. Neuroimage 31:968 980.

Düzel E, Bunzeck N, Guitart-Masip M, Wittmann B, Schott BH, Tobler PN (2009) Functional imaging of the human dopaminergic midbrain. Trends Neurosci 32:321-328.

Flodin P, Gospic K, Petrovic P, Fransson P (2012) Effects of L-DOPA and oxazepam on resting-state functional magnetic resonance imaging connectivity: a randomized, cross-sectional placebo study. Brain Connect 2:246-253.

Fonteneau C, Redoute J, Haesebaert F, Le Bars D, Costes N, Suaud-Chagny MF, Brunelin J (2018) Frontal transcranial direct current stimulation induces dopamine release in the ventral striatum in human. Cereb Cortex 28:2636-2646.

Fornai F, Chen K, Giorgi FS, Gesi M, Alessandri MG, Shih JC (1999) Striatal dopamine metabolism in monoamine oxidase B-deficient mice: a brain dialysis study. J Neurochem 73:2434-2440.

Foster HD, Hoffer A (2004) The two faces of L-DOPA: benefits and adverse side effects in the treatment of encephalitis lethargica, Parkinson's disease, multiple sclerosis and amyotrophic lateral sclerosis. Med Hypotheses 62: $177-181$.

Frankle WG, Laruelle M, Haber SN (2006) Prefrontal cortical projections to the midbrain in primates: evidence for a sparse connection. Neuropsychopharmacology 31:1627-1636.

Frazier JA, Chiu S, Breeze JL, Makris N, Lange N, Kennedy DN, Herbert MR, Bent EK, Koneru VK, Dieterich ME, Hodge SM, Rauch SL, Grant PE, Cohen BM, Seidman LJ, Caviness VS, Biederman J (2005) Structural brain magnetic resonance imaging of limbic and thalamic volumes in pediatric bipolar disorder. Am J Psychiatry 162:1256-1265.

Fryer SL, Roach BJ, Ford JM, Turner JA, van Erp TG, Voyvodic J, Preda A, Belger A, Bustillo J, O'Leary D, Mueller BA, Lim KO, McEwen SC, Calhoun VD, Diaz M, Glover G, Greve D, Wible CG, Vaidya J, Potkin SG, et al. (2015) Relating intrinsic low-frequency BOLD cortical oscillations to cognition in schizophrenia. Neuropsychopharmacology 40:2705-2714.

Galvan A, Wichmann T (2008) Pathophysiology of parkinsonism. Clin Neurophysiol 119:1459-1474.

Goldstein JM, Seidman LJ, Makris N, Ahern T, O’Brien LM, Caviness VS Jr, 
Kennedy DN, Faraone SV, Tsuang MT (2007) Hypothalamic abnormalities in schizophrenia: sex effects and genetic vulnerability. Biol Psychiatry 61:935-945.

Goto Y, Grace AA (2007) The dopamine system and the pathophysiology of schizophrenia: a basic science perspective. Int Rev Neurobiol 78:41-68.

Haaker J, Gaburro S, Sah A, Gartmann N, Lonsdorf TB, Meier K, Singewald N, Pape HC, Morellini F, Kalisch R (2013) Single dose of L-DOPA makes extinction memories context-independent and prevents the return of fear. Proc Natl Acad Sci U S A 110:E2428-E2436.

Haber SN, Knutson B (2010) The reward circuit: linking primate anatomy and human imaging. Neuropsychopharmacology 35:4-26.

Hawrylycz MJ, Lein ES, Guillozet-Bongaarts AL, Shen EH, Ng L, Miller JA, van de Lagemaat LN, Smith KA, Ebbert A, Riley ZL, Abajian C, Beckmann CF, Bernard A, Bertagnolli D, Boe AF, Cartagena PM, Chakravarty MM, Chapin M, Chong J, Dalley RA, et al. (2012) An anatomically comprehensive atlas of the adult human brain transcriptome. Nature 489: 391-399.

Hedreen JC, DeLong MR (1991) Organization of striatopallidal, striatonigral, and nigrostriatal projections in the macaque. J Comp Neurol 304:569-595.

Hilal-Dandan R, Brunton L (2014) Goodman and Gilman manual of pharmacology and therapeutics, Vol 2. Auflage. New York: McGraw-Hill.

Hone-Blanchet A, Edden RA, Fecteau S (2016) Online effects of transcranial direct current stimulation in real time on human prefrontal and striatal metabolites. Biol Psychiatry 80:432-438.

Kalivas PW, Volkow ND (2005) The neural basis of addiction: a pathology of motivation and choice. Am J Psychiatry 162:1403-1413.

Kalu UG, Sexton CE, Loo CK, Ebmeier KP (2012) Transcranial direct current stimulation in the treatment of major depression: a meta-analysis. Psychol Med 42:1791-1800.

Katzenschlager R, Lees AJ (2002) Treatment of Parkinson's disease: levodopa as the first choice. J Neurol 249 [Suppl 2]:II19-II24.

Leffa DT, de Souza A, Scarabelot VL, Medeiros LF, de Oliveira C, Grevet EH, Caumo W, de Souza DO, Rohde LA, Torres IL (2016) Transcranial direct current stimulation improves short-term memory in an animal model of attention-deficit/hyperactivity disorder. Eur Neuropsychopharmacol 26:368-377.

Liu CH, Liu CZ, Zhang J, Yuan Z, Tang LR, Tie CL, Fan J, Liu QQ (2016) Reduced spontaneous neuronal activity in the insular cortex and thalamus in healthy adults with insomnia symptoms. Brain Res 1648:317-324.

Lu C, Wei Y, Hu R, Wang Y, Li K, Li X (2015) Transcranial direct current stimulation ameliorates behavioral deficits and reduces oxidative stress in 1-methyl-4-phenyl-1,2,3,6-tetrahydropyridine-induced mouse model of Parkinson's disease. Neuromodulation 18:442-446; discussion 447.

Maandag NJ, Coman D, Sanganahalli BG, Herman P, Smith AJ, Blumenfeld H, Shulman RG, Hyder F (2007) Energetics of neuronal signaling and fMRI activity. Proc Natl Acad Sci U S A 104:20546-20551.

Makris N, Goldstein JM, Kennedy D, Hodge SM, Caviness VS, Faraone SV, Tsuang MT, Seidman LJ (2006) Decreased volume of left and total anterior insular lobule in schizophrenia. Schizophr Res 83:155-171.

Nitsche MA, Paulus W (2000) Excitability changes induced in the human motor cortex by weak transcranial direct current stimulation. J Physiol 527:633-639.

Polanía R, Paulus W, Nitsche MA (2012) Modulating cortico-striatal and thalamo-cortical functional connectivity with transcranial direct current stimulation. Hum Brain Mapp 33:2499-2508.
Power JD, Barnes KA, Snyder AZ, Schlaggar BL, Petersen SE (2012) Spurious but systematic correlations in functional connectivity MRI networks arise from subject motion. Neuroimage 59:2142-2154.

Rahman A, Reato D, Arlotti M, Gasca F, Datta A, Parra LC, Bikson M (2013) Cellular effects of acute direct current stimulation: somatic and synaptic terminal effects. J Physiol 591:2563-2578.

Sánchez MG, Morissette M, Di Paolo T (2012) Effect of a chronic treatment with $17 \beta$-estradiol on striatal dopamine neurotransmission and the Akt/ GSK3 signaling pathway in the brain of ovariectomized monkeys. Psychoneuroendocrinology 37:280-291.

Saturnino GB, Antunes A, Thielscher A (2015) On the importance of electrode parameters for shaping electric field patterns generated by tDCS. Neuroimage 120:25-35.

Schwalm M, Schmid F, Wachsmuth L, Backhaus H, Kronfeld A, Aedo Jury F, Prouvot PH, Fois C, Albers F, van Alst T, Faber C, Stroh A (2017) Cortex-wide BOLD fMRI activity reflects locally-recorded slow oscillation-associated calcium waves. eLife 6:e27602.

Takano Y, Yokawa T, Masuda A, Niimi J, Tanaka S, Hironaka N (2011) A rat model for measuring the effectiveness of transcranial direct current stimulation using fMRI. Neurosci Lett 491:40-43.

Thielscher A, Antunes A, Saturnino GB (2015) Field modeling for transcranial magnetic stimulation: a useful tool to understand the physiological effects of TMS? Conf Proc IEEE Eng Med Biol Soc 2015:222-225.

Valdés-Hernández PA, Sumiyoshi A, Nonaka H, Haga R, Aubert-Vásquez E, Ogawa T, Iturria-Medina Y, Riera JJ, Kawashima R (2011) An in vivo MRI template set for morphometry, tissue segmentation, and fMRI localization in rats. Front Neuroinform 5:26.

van Dam WO, Decker SL, Durbin JS, Vendemia JM, Desai RH (2015) Resting state signatures of domain and demand-specific working memory performance. Neuroimage 118:174-182.

Volkow ND, Fowler JS, Gatley SJ, Logan J, Wang GJ, Ding YS, Dewey S (1996) PET evaluation of the dopamine system of the human brain. J Nucl Med 37:1242-1256.

Xu Y, Zhuo C, Qin W, Zhu J, Yu C (2015) Altered spontaneous brain activity in schizophrenia: a meta-analysis and a large-sample study. Biomed Res Int 2015:204628.

Yan CG, Rincón-Cortés M, Raineki C, Sarro E, Colcombe S, Guilfoyle DN, Yang Z, Gerum S, Biswal BB, Milham MP, Sullivan RM, Castellanos FX (2017) Aberrant development of intrinsic brain activity in a rat model of caregiver maltreatment of offspring. Transl Psychiatry 7:e1005.

Yarkoni T, Poldrack RA, Nichols TE, Van Essen DC, Wager TD (2011) Large-scale automated synthesis of human functional neuroimaging data. Nat Methods 8:665-670.

Zang YF, He Y, Zhu CZ, Cao QJ, Sui MQ, Liang M, Tian LX, Jiang TZ, Wang YF (2007) Altered baseline brain activity in children with ADHD revealed by resting-state functional MRI. Brain Dev 29:83-91.

Zhou Y, Yu F, Duong TQ (2015) White matter lesion load is associated with resting state functional MRI activity and amyloid PET but not FDG in mild cognitive impairment and early Alzheimer's disease patients. J Magn Reson Imaging 41:102-109.

Zou QH, Zhu CZ, Yang Y, Zuo XN, Long XY, Cao QJ, Wang YF, Zang YF (2008) An improved approach to detection of amplitude of lowfrequency fluctuation (ALFF) for resting-state fMRI: fractional ALFF. J Neurosci Methods 172:137-141. 